\title{
Comparison of dexmedetomidine and propofol in mechanically ventilated patients with sepsis: A pilot study
}

\author{
Mark B. Sigler MD, Ebtesam A. Islam MD PhD, Kenneth M. Nugent MD
}

\begin{abstract}
Objective: To compare the effects of a propofol-based versus dexmedetomidine-based sedation regimen for mechanically ventilated patients with sepsis.

Methods: Single-center, randomized, open-label interventional study of critically ill patients admitted to the intensive care unit with sepsis and respiratory failure requiring mechanical ventilation. Patients were sedated with either propofol or dexmedetomidine.

Results: Thirty-six patients with sepsis and respiratory failure requiring mechanical ventilation were randomly assigned to receive sedation with either dexmedetomidine or propofol. Fentanyl was used for analgesia in both groups. The primary end point was duration of mechanical ventilation, and secondary end points included 28-day mortality, the duration of ICU stay, and the duration of vasopressor support. There was a non-statistically significant trend toward decreased duration of mechanical ventilation in the dexmedetomidine group $(p=0.107)$, and multivariable analysis demonstrated a small to moderate effect size in the sample. There were no significant differences in 28-day mortality, duration of ICU stay, or duration of vasopressor requirement. No patients required discontinuation of study drug due to adverse effects.

Conclusions: Although underpowered for statistical significance, there was a trend toward decreased duration of mechanical ventilation with dexmedetomidine. More studies with higher patient enrollment are needed to determine whether the duration of mechanical ventilation in patients with sepsis who receive sedation with dexmedetomidine is reduced when compared to propofol.
\end{abstract}

Keywords: sepsis, respiratory failure, ventilators, mechanical, propofol, dexmedetomidine, sedation

\section{INTRODUCTION}

Patients requiring mechanical ventilation often need sedation to maintain comfort. Historically, benzodiazepines and propofol were the preferred sedatives in the intensive care unit (ICU), but dexmedetomidine has recently had increased usage. ${ }^{1-3}$ Benzodiazepines and propofol primarily act at the

Corresponding author: Mark Sigler Contact Information: Sigler.mark@gmail.com DOI: 10.12746/swrccc.v6i22.444
$\mathrm{GABA}_{\mathrm{A}}$ receptor; dexmedetomidine is an imidazole compound that is a specific $\alpha_{2}$-adrenoceptor agonist. Although the mechanism of action of dexmedetomidine is not completely understood, the primary mechanism likely involves presynaptic activation of the $\alpha_{2}$-adrenoceptor and subsequent inhibition of the release of norepinephrine and termination of pain signal propagation. Additionally, post-synaptic activation of $\alpha_{2}$-adrenoceptors in the central nervous system inhibits sympathetic activity, resulting in decreased blood pressures and heart rates. ${ }^{4}$ This combination of effects results in analgesia, sedation, and anxiolysis, and patients sedated with dexmedetomidine 
are more arousable and have minimal respiratory depression. ${ }^{5}$

Since its initial approval by the FDA in 1999 for sedation of intubated and mechanically ventilated patients in the ICU, dexmedetomidine has been extensively studied in well-designed, randomized trials in comparison to other sedative drugs. In 2007, the MENDS trial compared dexmedetomidine to lorazepam and found that dexmedetomidine was associated with less delirium and more time spent at the targeted level of sedation. ${ }^{6}$ The SEDCOM trial in 2009 compared dexmedetomidine to midazolam and demonstrated a reduced duration of mechanical ventilation and less delirium in patients sedated with dexmedetomidine. ${ }^{7}$ The PRODEX and MIDEX trials were conducted jointly and compared dexmedetomidine to propofol and midazolam, respectively. These trials showed a significantly decreased duration of mechanical ventilation with dexmedetomidine compared to midazolam, and a non-significant decrease in the duration of mechanical ventilation with dexmedetomidine compared to propofol. ${ }^{8}$ When the sepsis subgroup in the MENDS trial was reanalyzed, a significant mortality benefit was found for patients sedated with dexmedetomidine. ${ }^{9}$

Most sedatives, including propofol and benzodiazepines, suppress the innate immune response. ${ }^{10}$ In contrast, dexmedetomidine stimulates the innate immune response in animal studies. ${ }^{11,12}$ Immune potentiation with dexmedetomidine has been considered a possible contributing factor to the mortality benefit observed in septic patients sedated with dexmedetomidine rather than lorazepam, which is associated with immune suppression. In current practice, non-benzodiazepine sedation (with propofol or dexmedetomidine) is recommended due to the association of benzodiazepines with adverse clinical outcomes, including longer duration of mechanical ventilation, increased ICU length of stay, and the development of delirium. ${ }^{3,13,14}$ The PRODEX trial included a broad spectrum of patients requiring mechanical ventilation and did not find a statistically significant difference in duration of mechanical ventilation between dexmedetomidine and propofol. Thus, it is unknown whether patients with sepsis requiring mechanical ventilation will benefit from sedation with dexmedetomidine, and most centers do not consider one sedative superior to the other for patients in this group.

Since current data suggest a possible benefit with dexmedetomidine for sedation of mechanically ventilated patients with sepsis and current guidelines recommend sedation with either dexmedetomidine or propofol for mechanically ventilated patients, we designed the PRO-DEFENSE (Propofol versus Dexmedetomidine for Mechanically Ventilated Patients with Sepsis) to test the hypothesis that dexmedetomidine, when compared with propofol, reduces the duration of mechanical ventilation.

\section{Materials and Methods}

This prospective, open-label, randomized trial was conducted in the ICUs at University Medical Center in Lubbock, TX, between September 2014 and February 2016. The protocol was approved by the Institutional Review Board of the study center, and all patients or legally authorized representatives provided written informed consent. The study was designed by the investigators. Data were collected by the investigators and analyzed by the Clinical Research Institute at Texas Tech University Health Sciences Center in Lubbock, TX.

Eligible patients were age 18 to 89 years old, had a diagnosis of sepsis, and required mechanical ventilation. Sepsis was defined as a potential source of infection with $\geq 2$ of the following criteria: 1 ) temperature $<36.0^{\circ} \mathrm{C}$ or $>38.0^{\circ} \mathrm{C}$; 2) heart rate $>90 /$ minute; 3) respiratory rate $>20 /$ minute or $\mathrm{PaCO}_{2}<32 \mathrm{mmHg}$; 4) WBC count $<4000 / \mu \mathrm{L},>12000 / \mu \mathrm{L}$, or $>10 \%$ bands. Exclusion criteria included the following patients: 1) documented allergies to propofol, dexmedetomidine, fentanyl, eggs or egg products, or soy or soy products; 2) heart rates less than 50 /minute or grade 2 or 3 AV heart block; 3) mean arterial pressures less than $55 \mathrm{mmHg}$ despite fluid resuscitation and vasopressor support; 4) triglyceride levels $>400 \mathrm{mg} / \mathrm{dL}$.

Patients who met criteria were randomized into either a dexmedetomidine treatment group or a propofol treatment group on a 1:1 basis using a random 
number generator and sealed envelopes. Due to the different appearances of propofol (an emulsion) and dexmedetomidine, the study was not blinded. Detailed information regarding sedative and analgesic therapy prior to initiation of study drug, baseline demographics, and severity of illness were obtained at the time of enrollment after consent was signed.

After randomization, sedatives used before study enrollment were titrated off, and titration of the study drug was initiated. Fentanyl was used for analgesia for both study groups. Propofol was initiated at $5 \mathrm{mcg} / \mathrm{kg} /$ minute and titrated every 5 minutes by $5 \mathrm{mcg} / \mathrm{kg} /$ minute. The maximum dose of propofol was $80 \mathrm{mcg} / \mathrm{kg} / \mathrm{minute}$. Dexmedetomidine was initiated at $0.2 \mathrm{mcg} / \mathrm{kg} / \mathrm{hour}$ and titrated every 5 minutes by $0.1 \mathrm{mcg} / \mathrm{kg} / \mathrm{hour}$ to a maximum dose of $1.4 \mathrm{mcg} /$ $\mathrm{kg} /$ hour. The Richmond Agitated and Sedation Scale (RASS) target was -1 to +1 . Patients with inadequate sedation scores on their assigned drug received supplemental sedation with midazolam or lorazepam as needed based on nursing and physician assessment.

The primary end point was duration of mechanical ventilation. Secondary end points included the duration of ICU stay, the duration of vasopressor support, a composite outcome (number of days of ventilator support plus number of ICU days plus number of days of vasopressor support), the percent alive at discharge, transfer, or at 28 days of hospitalization, the number of patients who needed a second sedative in addition to their study drug, and the number of patients who required discontinuation of their sedation medication due to unacceptable side effects.

The median duration of ventilator support and interquartile range for each study group were calculated, and the two study groups were compared using non-parametric testing with the Mann Whitney $U$ test. Similar calculations for made for the length of ICU stay, duration of vasopressor support, and the composite score. The number of survivors in the two groups was tabulated and reported in terms of frequency and relative percentages. A chi-square test was used to compare the two study groups. Similar calculations were made for the number of patients needing supplemental sedation and for the number of patients requiring their study drug to be discontinued. $P$ values $\leq 0.05$ were considered statistically significant. Assuming an equal sample size allocation with $5 \%(\alpha=0.05)$ level of significance and $80 \%$ power, a total sample size of 260 (130 per study group) was needed to calculate a $35 \%$ reduction in duration of mechanical ventilation. Assuming a $20 \%$ attrition rate due to mortality, the figure was adjusted to 163 individuals in each study group (total of 326 patients). This analysis was based on non-parametric testing with the Mann-WhitneyWilcoxon test. An interim analysis was planned after the first 100 patients had been enrolled in the study.

\section{RESULTS}

From September 9, 2014, through March 1, 2016 , 42 patients with respiratory failure requiring mechanical ventilation and sepsis were enrolled in the PRODEFENSE study. Six patients were subsequently excluded, and 36 were included in the analysis: 19 in the propofol group and 17 in the dexmedetomidine group. Due to the slow enrollment, an interim analysis was conducted in March 2016, and the study investigators decided to discontinue the trial.

The characteristics of the patients at inclusion in the study were similar in the two groups (Table 1). The sources for infection included the respiratory tract $(n=31)$, urinary tract $(n=7)$, abdomen $(n=3)$, cellulitis $(n=2)$, and miscellaneous $(n=4)$ (several patients had more than one source). The $\mathrm{PaO}_{2}: \mathrm{FiO}_{2}$ ratio was 205 \pm 166 in the propofol group and $200 \pm 62$ in the dexmedetomidine group. Because the attrition rate was higher than expected, a preliminary analysis was conducted using the non-parametric Mann-Whitney $U$ test to compare continuous covariates and Fisher's Exact test to compare categorical covariates. Baseline covariates were compared to identify any clinical differences between groups (Table 2). The time from intubation to randomization (3.0; interquartile range [IQR]: 6.4 hours for the dexmedetomidine group vs. 10.0; IQR: 18.3 for the propofol group, $p=0.010$ ) and the use of vasopressors $(82.4 \%$ for dexmedetomidine group vs. $47.4 \%$ for propofol group, $p=0.041$ ) were the only statistically significant differences between the two groups.

In univariate analysis, the primary outcome (number of ventilator days) was shorter in the 
Table 1: Baseline characteristics of patients

\begin{tabular}{|c|c|c|c|c|c|}
\hline \multirow[b]{2}{*}{ Parametric covariates } & \multicolumn{2}{|c|}{ Dexmedetomidine $(n=17)$} & \multicolumn{2}{|c|}{ Propofol $(n=19)$} & \multirow[b]{2}{*}{ p-value } \\
\hline & Mean & SD & Mean & SD & \\
\hline Age & 62.5 & 9.6 & 59.0 & 15.4 & 0.419 \\
\hline BMI & 29.7 & 8.2 & 30.1 & 6.5 & 0.824 \\
\hline PEEP at $24 \mathrm{hrs}$. & 6.0 & 1.8 & 7.2 & 3.0 & 0.253 \\
\hline $\mathrm{PaO}_{2}: \mathrm{FiO}_{2}$ ratio at $24 \mathrm{hrs}$. & 195 & 76 & 210 & 85 & 0.626 \\
\hline Ordinal covariates & Median & $(\mathbf{Q} 1, \mathrm{Q3})$ & Median & $(\mathrm{Q} 1, \mathrm{Q3})$ & p-value \\
\hline SOFA score & 11 & $(7,14)$ & 10 & $(8,13)$ & 0.924 \\
\hline APACHE2 score & 19 & $(13,20)$ & 16.0 & $(12,19)$ & 0.349 \\
\hline Categorical covariates & Count & $\%$ & Count & $\%$ & p-value \\
\hline Male & 13 & $76 \%$ & 9 & $47 \%$ & 0.097 \\
\hline White & 15 & $88 \%$ & 19 & $100 \%$ & 0.216 \\
\hline Hispanic & 5 & $29 \%$ & 7 & $37 \%$ & 0.732 \\
\hline Transfer patients & 7 & $41 \%$ & 11 & $58 \%$ & 0.505 \\
\hline
\end{tabular}

dexmedetomidine group, but this did not reach statistical significance $(p=0.107)$ (Table 3$)$. The number of ICU days and the number of vasopressor days were similar in the two groups $(p>0.25)$. The twenty-eight day mortality was $42.1 \%(8 / 19)$ in the propofol group and $52.9 \%(9 / 17)$ in the dexmedetomidine group $(p=$ 0.249 ). Given the possible difference in ventilator days, an adjusted analysis was used to address potential differences between groups at baseline. This multivariable analysis adjusted for potential differences in age, BMI, and SOFA, and/or APACHE2 scores in the two study groups. All three competing models (SOFA, APACHE2, and SOFA and APACHE2) had similar $p$-values $(p=0.126, p=0.129, p=0.131)$, and no covariates were significant factors $(p>0.10)$. Based on this analysis, the estimated least-square mean difference between dexmedetomidine and propofol was 3.13 ventilator days $(95 \% \mathrm{Cl}:-7.23$ to 0.96 days, $p=0.129)$. While this result did not reach significance (alpha $>0.05)$, the partial eta-squared of $0.073(95 \%$

Table 2: Details of study drugs administered

\begin{tabular}{|c|c|c|c|c|c|}
\hline \multirow[b]{2}{*}{ Non-parametric covariates } & \multicolumn{2}{|c|}{ Dexmedetomidine $(n=17)$} & \multicolumn{2}{|c|}{ Propofol $(n=19)$} & \multirow[b]{2}{*}{ p-value } \\
\hline & Median & $(\mathrm{Q} 1, \mathrm{Q3})$ & Median & $(\mathbf{Q 1}, \mathrm{Q3})$ & \\
\hline Duration of study drug infusion (days) & $2.8^{*}$ & $(1.5,4.0)$ & $3.0 \dagger$ & $(1.3,4.0)$ & 0.603 \\
\hline Intubation to randomization (hrs) & 3.0 & $(1.8,8.25)$ & 10.0 & $(4.2,22.5)$ & 0.010 \\
\hline Total fentanyl received $(\mu \mathrm{g})$ & 290 & $(100,475)$ & 478.8 & $(184,1165)$ & 0.096 \\
\hline Categorical covariates & Count & $\%$ & Count & $\%$ & p-value \\
\hline Vasopressors used & 14 & $82 \%$ & 9 & $47 \%$ & 0.041 \\
\hline Supplemental sedation & 0 & $0.0 \%$ & 0 & $0.0 \%$ & 1.000 \\
\hline Adverse events from study drug & 0 & $0.0 \%$ & 0 & $0.0 \%$ & 1.000 \\
\hline
\end{tabular}

* 2 missing values

$\dagger 4$ missing values 
Table 3: Study outcomes

\begin{tabular}{|l|c|c|c|c|c|}
\hline \multirow{2}{*}{ Continuous outcomes } & \multicolumn{2}{|c|}{ Dexmedetomidine $(\mathbf{n}=\mathbf{1 7})$} & \multicolumn{2}{c|}{ Propofol (n= 19) } & \multirow{2}{*}{ p-value } \\
\cline { 2 - 5 } & Median & IQR (Q3-Q1) & Median & IQR (Q3-Q1) & 0.107 \\
\hline Days of mechanical ventilation & 3 & $(2.75,5.75)$ & 5 & $(3,13)$ & 0.260 \\
\hline Days of ICU stay & 5 & $(3,8)$ & 6 & $(4,14)$ & 0.376 \\
\hline Days of vasopressor infusion* & 2 & $(0,3)$ & 0 & $(0,3.3)$ & 0.202 \\
\hline Composite outcome* (sum the above) & 10.5 & $(5.0,18.25)$ & 11.8 & $(9.8,28.0)$ & p-value \\
\hline Categorical outcomes & Count & $\mathbf{\%}$ & Count & \% & 0.739 \\
\hline Mortality (28 days) & 9 & $53 \%$ & 8 & $42 \%$ & \\
\hline
\end{tabular}

*one outcome was missing value from each of the two groups.

Cl: 0-0.259) corresponds to a small to moderate effect size in this sample. A power analysis suggested that 64 subjects in each group would be sufficient to probe an alpha of 0.05 at $80 \%$ power.

\section{Discussion}

In this single center, randomized trial comparing dexmedetomidine and propofol for sedation in mechanically ventilated patients with sepsis, there was no statistically significant difference in the duration of mechanical ventilation between patients who received dexmedetomidine and those who received propofol. There was a non-statistically significant trend toward decreased duration of mechanical ventilation in the dexmedetomidine group, and this trend remained after multivariate analysis. Secondary outcomes, including mortality, ICU days, and vasopressor days, were not different between the two groups. In the 36 patients who underwent randomization, no patients required discontinuation of the assigned sedative medication secondary to adverse effects.

This study was designed to determine whether the subset of mechanically ventilated patients with sepsis would have a difference in mechanical ventilation with dexmedetomidine compared with propofol. Although underpowered, the available data from our study suggest that mechanically ventilated patients with sepsis may have a decreased duration of mechanical ventilation when sedated with dexmedetomidine. Despite an increased severity of illness in the dexmedetomidine group, this trend toward decreased duration of mechanical ventilation with dexmedetomidine remained after adjusted analysis.

Klompas, et al. recently evaluated dexmedetomidine, propofol, and benzodiazepines as sedatives in mechanically ventilated patients in a meta-analysis and noted a decreased time to extubation with dexmedetomidine compared to propofol and benzodiazepines and a trend toward a decreased incidence of ventilator-associated events with dexmedetomidine compared to propofol and benzodiazepines. ${ }^{15}$ One limitation in this meta-analysis was the reduced use of dexmedetomidine compared to other sedative medications. However, this study provides additional evidence in support of an association between decreased duration of mechanical ventilation and sedation with dexmedetomidine. This analysis by Klompas did not demonstrate any differences in mortality.

Our trial has several limitations. First, enrollment was limited, which resulted in the study being underpowered to detect statistically significant differences. Differences in comorbidity in the two groups could also have influenced outcomes. There were differences between the two groups in the baseline requirement for vasopressors. However, this was factored in to the adjusted analysis. Second, this was a single center study, and therefore our results may not be generalizable to other institutions. Third, due to the technical difficulties with concealing propofol and dexmedetomidine infusions, our study was not blinded. Despite these limitations, this trial does reveal some clinically 
important findings. First, both dexmedetomidine and propofol appear safe for use as sedatives in mechanically ventilated patients with sepsis. In this trial, no adverse effects requiring study drug discontinuation occurred. Second, despite being underpowered, there was a trend toward decreased duration of mechanical ventilation with dexmedetomidine, and this trend persisted after multivariable analysis. When interpreted in reference to recent meta-analysis by Klompas, this result emphasizes the potential importance of more studies comparing dexmedetomidine and propofol, particularly in patients with sepsis.

\section{Conclusions}

Both dexmedetomidine and propofol appear to be safe sedatives in mechanically ventilated patients with sepsis. This study suggests a possible decrease in duration of mechanical ventilation in patients sedated with dexmedetomidine, but additional studies are needed to determine whether this is statistically significant.

Acknowledgements: The authors wish to acknowledge the contribution of the Texas Tech University Health Sciences Center Clinical Research Institute for its assistance with this research and its analysis.

Article citation: Sigler MB, Islam EA, Nugent K. Comparison of dexmedetomidine and propofol in mechanically ventilated patients with sepsis: a pilot study. The Southwest Respiratory and Critical Care Chronicles 2018;6(22):10-15.

From: Department of Internal Medicine, Texas Tech University Health Sciences Center, Lubbock, Texas

Submitted: 9/1/2917

Accepted: $1 / 10 / 2018$

Reviewers: Randall Rosenblatt MD

Conflicts of interest: none

\section{REFERENCES}

1. Wunsch H, Kahn JM, Kramer AA, et al. Use of intravenous infusion sedation among mechanically ventilated patients in the United States. Crit Care Med 2009;37:3031-3039.
2. Tan JA, Ho KM: Use of dexmedetomidine as a sedative and analgesic agent in critically ill adult patients: A metaanalysis. Intensive Care Med 2010;36:926-939.

3. Barr J, Fraser GL, Puntillo K, et al. Clinical Practice Guidelines for the Management of Pain, Agitation, and Delirium in Adult Patients in the Intensive Care Unit. Crit Care Med 2013;41:263-306.

4. Gertler R, Brown HC, Mitchell DH, et al. Dexmedetomidine: a novel sedative-analgesic agent. BUMC Proceedings 2001;14:13-21.

5. Belleville JP, Ward DS, Bloor BC, et al: Effects of intravenous dexmedetomidine in humans. I. Sedation, ventilation, and metabolic rate. Anesthesiology 1992;77:1125-1133.

6. Pandharipande PP, Pun BT, Herr DL, et al. Effect of sedation with dexmedetomidine vs lorazepam on acute brain dysfunction in mechanically ventilated patients. JAMA 2007;298: 2655-2653.

7. Riker RR, Shehabi Y, Bokesch PM, et al. Dexmedetomidine vs midazolam for sedation of critically ill patients. JAMA 2009;301:489-499.

8. Jakob SM, Ruokonen E, Grounds RM, et al. Dexmedetomidine vs midazolam or propofol for sedation during prolonged mechanical ventilation. JAMA 2012;307:1151-1160.

9. Pandharipande PP, Sanders RD, Girard TD. Effect of dexmedetomidine versus lorazepam on outcome in patients with sepsis: an a priori-designed analysis of the MENDS randomized controlled trial. Critical Care 2010;14:R38.

10. Sanders RD, Hussell T, Maze M. Sedation \& Immunomodulation. Crit Care Clin 2009;25(551-570).

11. Taniguchi T, Kidani $Y$, Kanakura H, et al. Effects of dexmedetomidine on mortality rate and inflammatory responses to endotoxin-induced shock in rats. Crit Care Med 2004;32: 1322-1326.

12. Taniguchi T, Kurita A, Kobayashi K, et al. Dose- and time-related effects of dexmedetomidine on mortality and inflammatory responses to endotoxin-induced shock in rats. J Anesth 2008;22:221-228.

13. Fong JJ, Kanji S, Dasta JF, et al. Propofol associated with a shorter duration of mechanical ventilation than scheduled intermittent lorazepam: A database analysis using Project IMPACT. Ann Pharmacother 2007;41:1986-1991.

14. Esmaoglu A, Ulgey A, Akin A, et al. Comparison between dexmedetomidine and midazolam for sedation of eclampsia patients in the intensive care unit. J Crit Care 2009;24:551-555.

15. Klompas M, Lingling L, Szumita $P$, et al. Associations between different sedatives and ventilator-associated events, length of stay, and mortality in patients who were mechanically ventilated. Chest 2016;149:1373-1379. 